\title{
The Epipeptide Biosynthesis Locus epeXEPAB Is Widely Distributed in Firmicutes and Triggers Intrinsic Cell Envelope Stress
}

\author{
Philipp F. Popp ${ }^{a, b} \quad$ Lena Friebel $^{a} \quad$ Alhosna Benjdiac $^{c} \quad$ Alain Guillot $^{c}$ \\ Olivier Berteau ${ }^{c}$ Thorsten Mascher ${ }^{a}$ \\ anstitute of Microbiology, Technische Universität (TU) Dresden, Dresden, Germany; ${ }^{b}$ Institute for Biology - Bacterial \\ Physiology, Humboldt-Universität zu Berlin, Berlin, Germany; 'Université Paris-Saclay, INRAE, AgroParisTech, Micalis \\ Institute, ChemSyBio, Jouy-en-Josas, Jouy-en-Josas, France
}

\section{Keywords}

Bacillus subtilis - Comparative genomics - Antimicrobial peptide $\cdot$ Cell envelope stress response (3-5)

\begin{abstract}
The epeXEPAB (formerly yydFGHIJ) locus of Bacillus subtilis encodes a minimalistic biosynthetic pathway for a linear antimicrobial epipeptide, EpeX, which is ribosomally produced and post-translationally processed by the action of the radical-SAM epimerase, EpeE, and a membrane-anchored signal 2 peptide peptidase, EpeP. The $A B C$ transporter EpeAB provides intrinsic immunity against self-produced EpeX, without conferring resistance against extrinsically added EpeX. EpeX specifically targets, and severely perturbs the integrity of the cytoplasmic membrane, which leads to the induction of the Lia-dependent envelope stress response. Here, we provide new insights into the distribution, expression, and regulation of the minimalistic epeXEPAB locus of $B$. subtilis, as well as the biosynthesis and biological efficiency of the produced epipeptide EpeX*. A comprehensive comparative genomics study demonstrates that the epe-locus is restricted to but widely distributed within the phylum Firmicutes. The gene products of epeXEP are necessary and sufficient for the production of the mature antimicrobial peptide EpeX*. In $B$.
\end{abstract}

subtilis, the epeXEPAB locus is transcribed from three different promoters, one upstream of epeX $\left(\mathrm{P}_{\text {epeX }}\right)$ and two within epeP $\left(\mathrm{P}_{\text {epeA1 } 1}\right.$ and $\left.\mathrm{P}_{\text {epeA2 }}\right)$. While the latter two are mostly constitutive, $\mathrm{P}_{\text {epex }}$ shows a growth phase-dependent induction at the onset of stationary phase. We demonstrate that this regulation is the result of the antagonistic action of two global regulators: The transition state regulator AbrB keeps the epe locus shut off during exponential growth by direct binding. This tight repression is relieved by the master regulator of sporulation, Spo0A, which counteracts the AbrB-dependent repression of epeXEPAB expression during the transition to stationary phase. The net result of these three promoters is an expression pattern that ensures EpeAB-dependent autoimmunity prior to EpeX* production. In the absence of EpeAB, the general envelope stress response proteins LialH can compensate for the loss of specific autoimmunity by providing sufficient protection against the membrane-perturbating action of EpeX*. Hence, the transcriptional regulation of epe expression and the resulting intrinsic induction of the two corresponding resistance functions, encoded by epeAB and $I i a l H$, are well balanced to provide a need-based immunity against mature EpeX*.

(c) 2021 The Author(s)

Published by S. Karger AG, Basel karger@karger.com www.karger.com/mip

Karger $\stackrel{\text { ' }}{=}$
(C) 2021 The Author(s)

Published by S. Karger AG, Basel

This is an Open Access article licensed under the Creative Common Attribution-NonCommercial-4.0 International License (CC BY-NC) (http://www.karger.com/Services/OpenAccessLicense), applicable to the online version of the article only. Usage and distribution for commercial purposes requires written permission.
Correspondence to:

Thorsten Mascher, thorsten.mascher@tu-dresden.de 


\section{Introduction}

Bacteria thrive in a highly challenging, ever-changing world, where they need to establish themselves in the presence of competitors aiming for the same ecological niches. The production of and resistance against antimicrobial compounds represents one important aspect of this competition. It provides bacteria with a biochemical arsenal to suppress the growth of competitors, while simultaneously offering defense against compounds produced by them [Czárán et al., 2002; Foster and Bell, 2012; Kerr et al., 2002].

In Gram-positive bacteria, antimicrobial peptides (AMPs) represent a major compound class in this biological warfare. AMPs can either be ribosomally synthesized and post-translationally modified peptides (RiPPs or bacteriocins) or non-ribosomally synthesized peptide antibiotics. While the latter comprise a chemically highly diverse group of non-related compounds that share a common biosynthetic logic based on non-ribosomal peptide synthetases [Finking and Marahiel, 2004; Strieker et al., 2010], bacteriocins are chemically more coherent and are subdivided into three major classes: Class I bacteriocins are mostly heavily modified lanthioninecontaining lantibiotics (such as nisin or mersacidin), while Class II comprises the non-lanthionine-containing bacteriocins, including pediocin or lactococcin. In contrast to these two classes of small (less than 50 amino acids for the pre-pro-peptide), mostly heat-stable AMPs, Class III contains significantly larger, and hence heatlabile, AMPs such as colicin [Cotter et al., 2005; Kumariya et al., 2019].

Despite the chemical diversity of ribosomally synthesized AMPs, a number of common principles can be extracted for their biosynthetic pathways: (i) all ribosomally synthesized AMPs are encoded as larger prepro-peptides. (ii) The initial translation product often gets modified inside the cytoplasm. (iii) The modified pre-peptide then gets exported. (iv) During transport, the modified pre-peptide is usually further processed, e.g., by proteolysis of an $\mathrm{N}$-terminal signal peptide, to release the mature and active AMP into the environment. (v) The producing organism needs to be provided with immunity against this active AMP. These biosynthetic principles require the following functions to be encoded in AMP biosynthesis loci: (i) the structural gene of the AMP, (ii) modification enzymes, (iii) AMP exporters, (iv) membrane-anchored or extracellular peptidases that cleave the AMP during/after transport, and (v) auto-immunity functions. A tight regulation of the expression of the corresponding biosynthetic loci, usually at the transcriptional level, ensures a proper temporal coordination of AMP biosynthesis with autoimmunity.

All of these prerequisites also need to be fulfilled by a new AMP class that has recently been described in Bacillus subtilis: so-called epipeptides [Benjdia et al., 2017a; Benjdia et al., 2017b; Popp et al., 2020]. The corresponding AMP is encoded by epeX as a pre-pro-peptide of 49 amino acids. Post-translational modifications of two amino acids by the radical-SAM epimerase EpeE (formerly YydG) convert two amino acids, L-Val and L-Ile, to D-valine and D-allo-isoleucine, respectively (Fig. 1 [Benjdia et al., 2017a; Benjdia et al., 2017b];). The pre-peptide (a modified 49-mer) is further processed and exported to release the active form, EpeX* (formerly YydF*), as a linear peptide of 17 amino acids with two modified positions $\left(\mathrm{Val}_{4}\right.$ and $\left.\mathrm{Ile}_{12}\right)$. Based on the genetic architecture of the biosynthetic locus, both functions are predicted to be catalyzed simultaneously by EpeP, a putative membrane-anchored signal peptide peptidase (Fig. 1). In addition to these core biosynthetic functions, encoded by epeXEP, the locus only encodes the $A B C$ transporter EpeAB, which has been implicated in autoimmunity against EpeX [Butcher and Helmann, 2006] without conferring resistance against externally added EpeX* [Popp et al., 2020]. Based on its chemistry, the active RiPP EpeX* belongs to the Class II bacteriocins, but does not fit into any of the current sub-classes (IIa-IId). It is particularly active against $B$. subtilis but also other closely related Grampositive bacteria [Benjdia et al., 2017a]. EpeX* severely damages the cytoplasmic membrane by dissipating the membrane potential via membrane permeabilization, accompanied by a rapid reduction of membrane fluidity and lipid domain formation [Popp et al., 2020]. In B. subtilis, this massive attack on membrane integrity triggers a highly specific and narrow stress response, primarily activating the Lia system. This leads to the induction of the liaIH operon, which encodes a resistance determinant against extrinsically applied EpeX* [Popp et al., 2020].

Here, we address a number of open questions regarding the expression of the epeXEPAB locus, the biosynthesis of EpeX* and the resulting intrinsic stress response of $B$. subtilis. This includes the phylogenetic distribution of the epe-locus, the activity and regulation of the three promoters responsible for epeXEPAB expression, as well as the function of the signal peptide peptidase EpeP. 


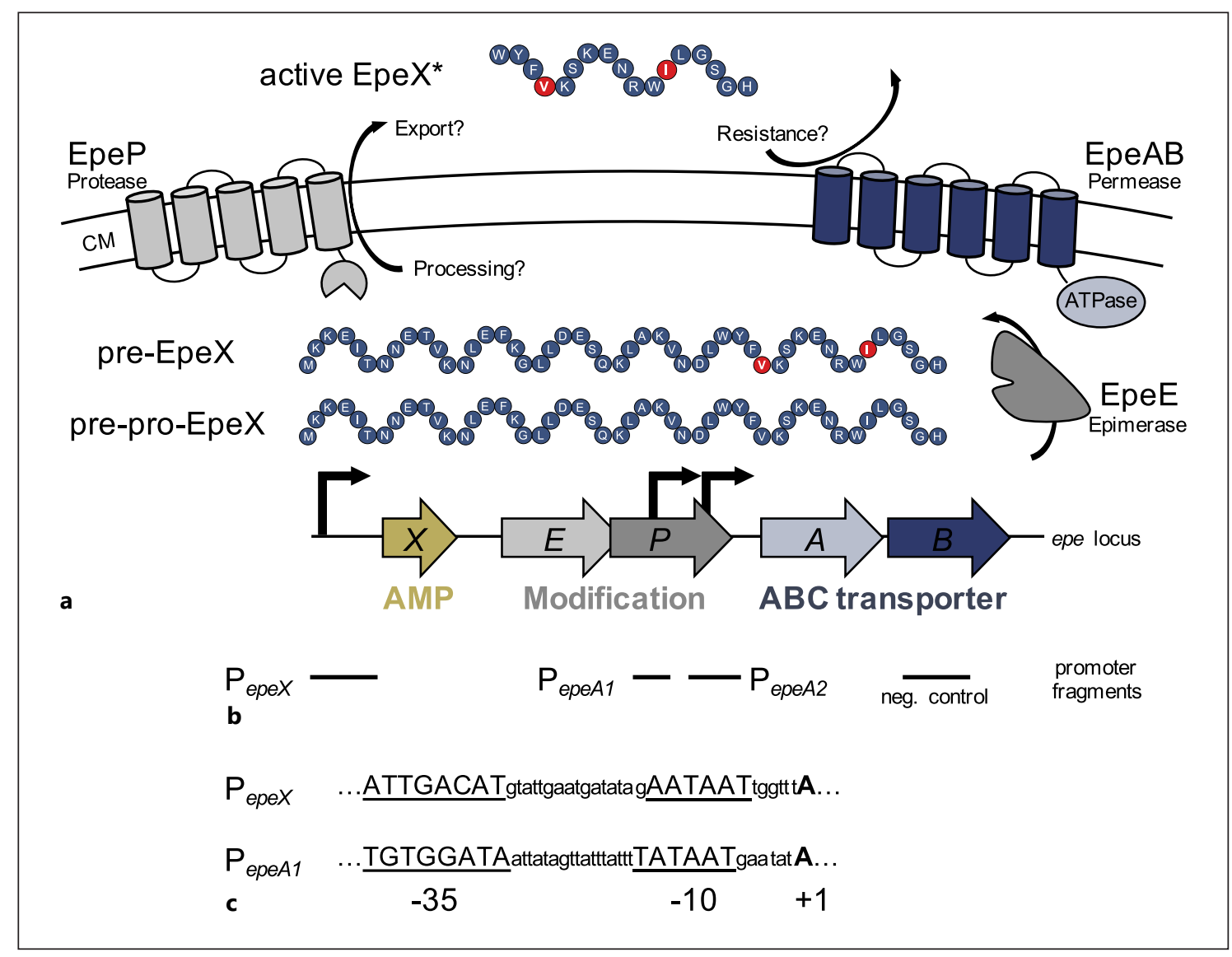

Fig. 1. Schematic representation of the epe locus and EpeX* biosynthesis in B. subtilis. a Three promoters drive the expression of the epe locus. Post-translational modification of pre-pro-EpeX is mediated by the epimerase EpeE (dark gray). The pre-peptide is then presumably processed and exported by the membrane-bound protease EpeP (light gray), resulting in the extracellular release of the active form EpeX*. The EpeAB ABC transporter is postulated to be involved autoimmunity against the antimicrobial activity of EpeX*. The two amino acids epimerized by EpeE are highlighted in red. $\mathbf{b}$ Schematic promoter fragments analyzed in this study to

\section{Results}

\section{EpeX-Like AMPs and the epeXEPAB Locus Are}

Widely Distributed in Firmicutes Bacteria

Previous studies demonstrated that the post-translational modification mediated by the radical SAM-epimerase EpeE results in the conversion of two L-amino acids into their D-forms within the EpeX peptide sequence. This modification is crucial for the biological activity of EpeX* [Benjdia et al., 2017a; Benjdia et al., 2017b]. In B. subtilis, epeX is encoded in an operon comprising epeX$E P A B$ (formerly yydFGHIJ; Fig. 1). We first addressed the phylogenetic distribution of this minimal AMP locus by unravel the transcriptional regulation of the epe locus. The two promoter fragments shown in c covering $\mathrm{P}_{\text {epeX }}$ and $\mathrm{P}_{\text {epeAl }}$ are derived from mapped transcriptional start sites identified by comprehensive tiling array studies [Nicolas et al., 2012]. The second promoter, driving epeA $\left(\mathrm{P}_{\text {epeAz }}\right)$ is newly identified. The negative control fragment contains no annotated promoter at the respective position. c Promoter sequences containing the -35 and -10 boxes and the predicted +1 of the previously mapped promoters $\mathrm{P}_{\text {epeX }}$ and $\mathrm{P}_{\text {epeAl }}$ [Nicolas et al., 2012].

performing three layers of bioinformatics analyses on (i) the conservation of EpeX peptides across bacteria, (ii) the identification of conserved amino acid residues within the EpeX sequence, with special attention to those being epimerized for its biological activity, and (iii) the genetic architecture of the epe locus genes.

For the first two aims, we performed multiple rounds of psi-blastp searches using the EpeX pre-pro-peptide sequence of $B$. subtilis as query (Material and Methods). After three sequential rounds, we obtained 50 EpeX hits, which were all restricted to, but distributed broadly within the phylum Firmicutes, without any preference for any specific genus (suppl. Table 1; for all online suppl. mate- 


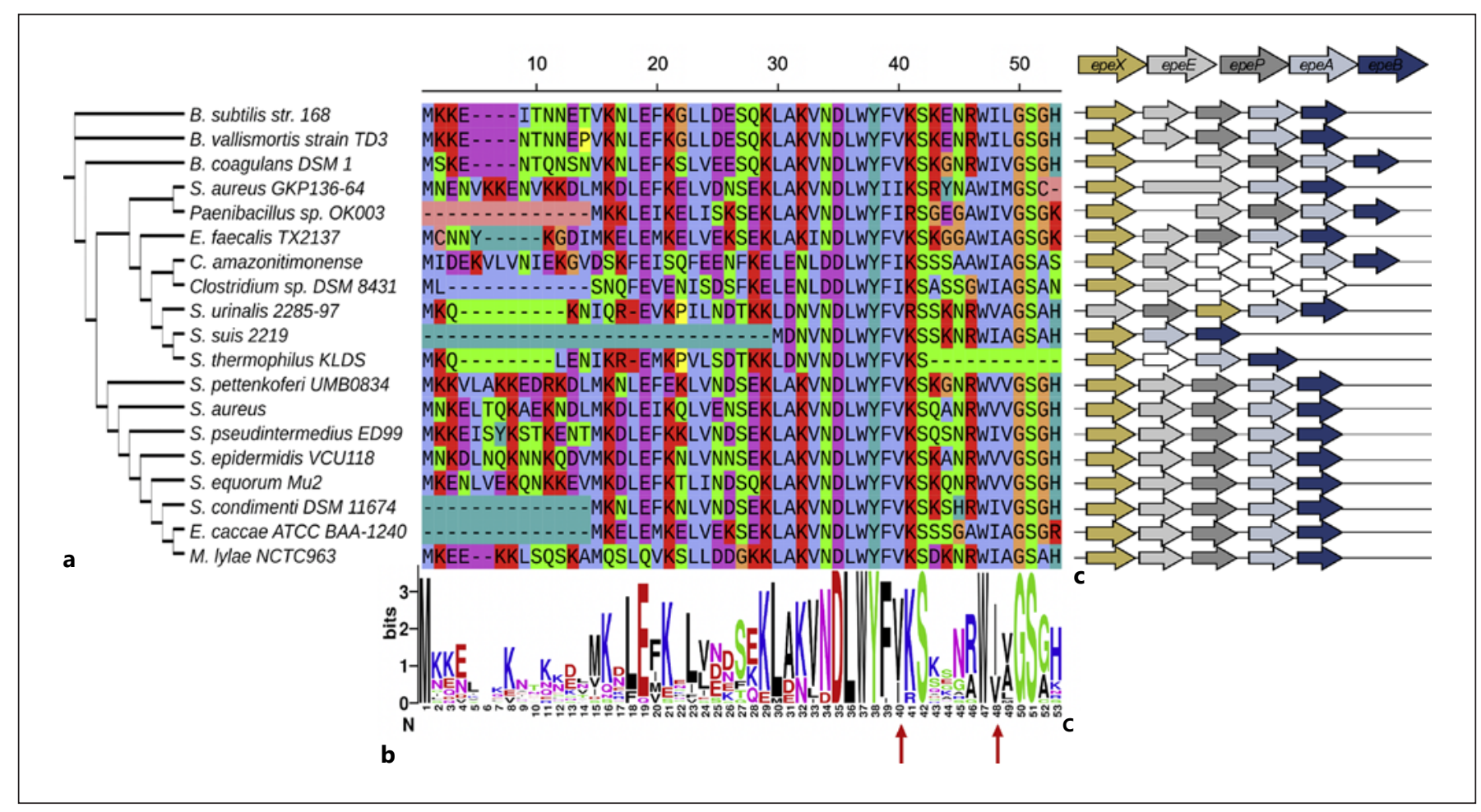

Fig. 2. Bioinformatics analysis of epe peptides from Firmicutes bacteria. a Maximum-likelihood phylogenetic tree of EpeX was computed using the build module in TREND, based on the respective peptide sequences derived from multiple rounds of psi-blastp [Gumerov, and Zhulin, 2020]. b Based on the multiple sequence alignment of EpeX peptides, the weblogo below [Crooks et al.,
2004] indicates regions of conservation. Red arrows highlight the amino acid residues that are epimerized from their L-form into the respective D-counterparts. c For each species, the genomic architecture of the epe locus is shown with the respective gene neighborhood on the right. White arrows indicate absence of epe association or hypothetical genes. For full dataset see suppl. Table 1. rial, see www.karger.com/doi/10.1159/000516750). After obtaining the peptide sequences of the psi-blastp search, we performed a multiple sequence alignment and generated a phylogenetic tree (shown in Fig. 2a and Material and Methods) to identify conserved regions within the EpeX sequence (Fig. 2b). Interestingly, the $\mathrm{N}$-terminal 17 amino acids of the pre-pro-peptide - which are lost during export - are only very weakly conserved. The second third of the EpeX sequence, amino acids 18-36, shows a moderate sequence conservation, indicating a potential recognition site for the corresponding membrane-bound EpeP peptidases also encoded within the locus, which could mediate export and processing of EpeX. Not surprisingly, the most prominent conservation was found in the C-terminal third, which encompasses the secreted and active form of the EpeX* peptide [Benjdia et al., 2017a]. Importantly, positions 40 and 48 of B. subtilis EpeX, which correspond to the epimerized amino acids, aligned either to valine or isoleucine in all EpeX orthologs. For the radical-SAM epimerase EpeE of B. subtilis, it was shown that both amino acids are accepted substrates for catalysis [Benjdia et al., 2017a], thus underscoring their functional relevance for EpeX* also in other species.

Next, we probed the gene neighborhood of each species in the dataset. Considering the minimalistic nature of the epeXEPAB locus of $B$. subtilis, we assumed that most genes should also be conserved in other species, which was indeed the case (Fig. 2c). Solely two members of the genus Streptococcus lack an epeE ortholog in the direct genomic vicinity. Our analysis also revealed that different gene arrangements of the epe locus are possible. In one strain of Staphylococcus urinalis, epeX is located in the middle of the operon rather than at its beginning (Fig. 2c). The $A B C$ transporter encoded by epe $A B$ is also highly conserved in most EpeX-encoding species, emphasizing its integral role for EpeX* immunity. In Clostridium sp. DSM 8431, only hypothetical genes are located next to epeXE (shown in Fig. 2c, white arrows). However, a detailed follow-up analysis revealed that a membrane- 

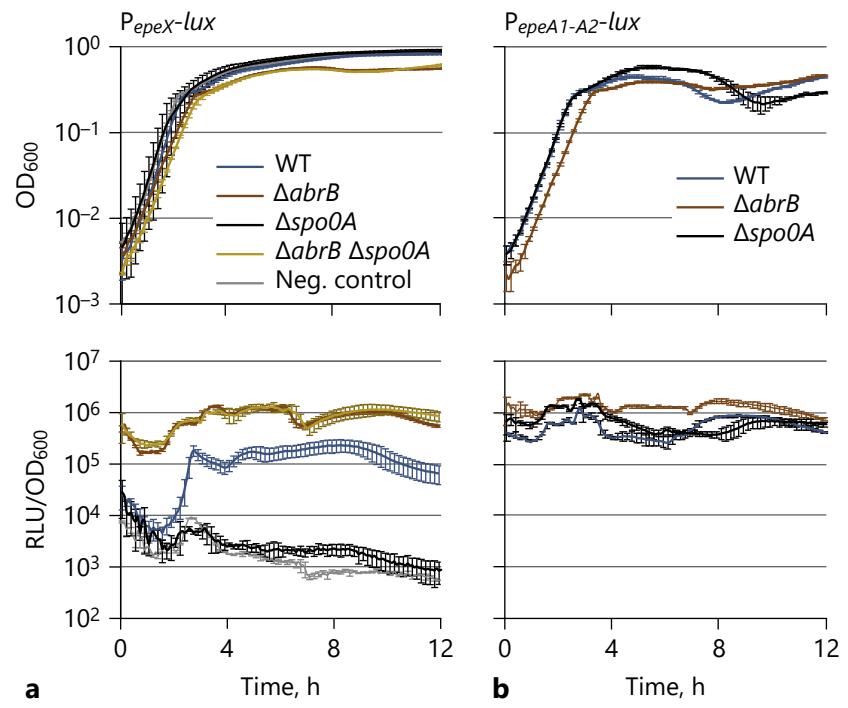
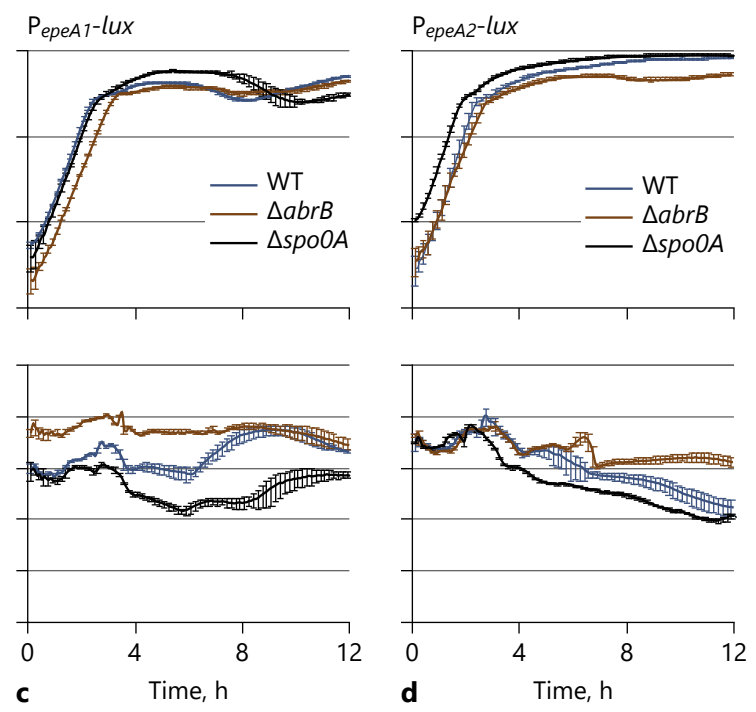

Fig. 3. Transcriptional regulation of the epe locus in B. subtilis. a $\mathrm{P}_{\text {epeX }}$ was placed upstream of the luxABCDE operon, and the construct was integrated into the sacA locus of $B$. subtilis. The $\mathrm{P}_{\text {epeX }}$ and the negative control fragments correspond to Fig. $1 \mathrm{~B}$. $\mathbf{b}$ The fragment covering $\mathrm{P}_{\text {epeA1 }}$ and $\mathrm{P}_{\text {epeA2 }}$ was placed upstream of $l u x$ $A B C D E$ operon, and its activity was monitored. c, d Both epeA

bound peptidase and an $\mathrm{ABC}$ transporter are encoded by them, thereby completing the epe locus.

Taken together, our bioinformatics analysis revealed a sporadic but wide distribution of the complete epeXEPAB locus in Firmicutes bacteria, without any phylogenetic preference for certain genera. If present, the C-terminal third of EpeX, including both epimerized amino acids, was well conserved, underscoring the functional relevance for the biological activity of EpeX*.

Expression of the epeXEPAB Locus in B. subtilis Is Growth Phase Dependent and under Direct Control of the Transition State Repressor AbrB

We next aimed at unraveling the expression and potential transcriptional regulation of this operon in B. subtilis. Comprehensive genome-wide transcriptional profiling indicated two putative promoters responsible for expressing the epeXEPAB locus, one in the intergenic region upstream of epeX $\left(\mathrm{P}_{\text {epeX }}\right)$ and a second transcriptional start site inside epeP $\left(\mathrm{P}_{\text {epeA1 }}\right)$, which could drive epeAB expression independent of $\mathrm{P}_{\text {epeX }}$ (suppl. Fig. S1) [Nicolas et al., 2012]. The promoters, as well as a negative control fragment derived from within epeB (Fig. 1b), were cloned upstream of a luxABCDE cassette and the corresponding

promoter activities separately. Growth (upper panel) and luminescence signal normalized to growth at each timepoint (lower panel) was followed over time. Measurements were performed every $5 \mathrm{~min}$ for $12 \mathrm{~h}$. Mean and standard deviation are shown from biological duplicates and technical triplicates.

reporter constructs were introduced into the sacA locus to monitor luminescence signal over time (Fig. 3). For $\mathrm{P}_{\text {epeX }}$, the luminescence signal (normalized to $\mathrm{OD}_{600}$ ) of the cloned fragment showed strong induction of activity late in exponential to onset of stationary phase in the wild type (Fig. 3a). This observation not only demonstrated that we had indeed cloned the epeX promoter, but is also in line with the known induction of the vast majority of secondary metabolite clusters at the onset of stationary phase. This timing of antibiotic production is attributed to its role as a survival strategy when nutrients become scarce [Lopez et al., 2009; Popp and Mascher, 2019].

In B. subtilis, many antibiotic biosynthesis clusters are under the transcriptional control of the transition state regulator AbrB and thereby also linked to its adversary, the sporulation master regulator Spo0A [Lopez and Kolter, 2010; Lopez et al., 2009]. We therefore performed our transcriptional analysis not only in the wild type, but also in isogenic mutant strains lacking one or both of these key regulators. No significant growth defects were observed in nutrient-rich medium between the individual mutants and the wild type, with only the $a b r B$ mutant being slightly impaired during stationary phase (Fig. 3, upper panels). In contrast to the wild type, the $\mathrm{P}_{\text {epeX }}$ activity was significantly 
higher in the abrB mutant throughout growth, resulting in a less pronounced transition state induction between 2 and $3 \mathrm{~h}$ of the experiment. In contrast, deletion of $\mathrm{Abh}$, the AbrB paralog, had no effect on $\mathrm{P}_{\text {epeX }}$ activity (data not shown). This result is in line with AbrB's role as a repressor of stationary phase operons during exponential growth (Fig. 3a, lower panel). Deletion of spo0A resulted in the opposite effect, that is, a 30 -fold reduction of promoter activity, again in line with its role as a positive regulator for many stationary phase-related functions [Lopez et al., 2009] (Fig. 3a, lower panel). Since both regulators also repress each other, directly or indirectly, we also tested an $a b r B / s p o 0 A$ double mutant to identify the regulator directly affecting $\mathrm{P}_{\text {epeX }}$ activity. In the corresponding reporter strain, we observed a behavior identical to the $a b r B$ single mutant (Fig. 3a, lower panel). This result genetically demonstrates that $\mathrm{AbrB}$ is the direct regulator of $\mathrm{P}_{\text {epeX }}$, while Spo0A affects epe expression indirectly through the known repression of $\mathrm{AbrB}$ at the transition to stationary phase, thereby causing prevalence of steady AbrB levels throughout growth [Strauch et al., 1990]. Our results are in perfect agreement with data from a genome-wide high-resolution mapping of in vivo $\mathrm{AbrB}$ binding, which identified the epeXEPAB locus as one of the most prominent target sites, with high-affinity and highoccupancy binding of $\mathrm{AbrB}$ across the entire epe locus of $B$. subtilis (Fig. S2) [Chumsakul et al., 2013]. The high AbrB occupancy explains the dramatic Spo0A effect on $\mathrm{P}_{\text {epeX }}$ activity shown in Figure 3a: the epe locus remains completely blocked throughout growth if $\mathrm{AbrB}$ repression is not released by Spo0A. Thus, the epe locus is under direct transcriptional control of AbrB.

Following the activity of $\mathrm{P}_{\text {epeA }}$ under the same experimental conditions revealed a more complex regulation. First, we cloned a larger fragment $\left(\mathrm{P}_{\text {epeA1-A2 }}\right)$, which included the previously mapped promoter, $\mathrm{P}_{\text {epeAl }}$ (suppl. Fig. S1) [Nicolas et al., 2012] and most of the remaining epeP gene downstream. The luminescence activity of this fragment revealed a strong growth phase-independent constitutive promoter (Fig. 3b). Interestingly, deletion of $a b r B$ again resulted in an elevated promoter activity compared to the WT. A spo0A deletion had only minor impact on the promoter dynamics, in contrast to the results obtained with $\mathrm{P}_{\text {epeX }}$ (Fig. 3a). Subsequently, we split this large epeP-internal fragment into two, one only containing the previously mapped $\mathrm{P}_{\text {epeA1 }}$ region, while the second - and larger - fragment covered the remaining $3^{\prime}$ region directly downstream of $\mathrm{P}_{\text {epeA1 }}$ (here termed $\mathrm{P}_{\text {epeA } 2}$ ). The $\mathrm{P}_{\text {epeAl }}$ fragment showed slight induction of promoter activity in late stationary phase in the WT (Fig. 3c). In the abrB mutant, $\mathrm{P}_{\text {epeAl }}$ promoter activity remained elevated throughout stationary

Epipeptide Biosynthesis Locus of $B$. subtilis phase, indicating that the massive AbrB binding observed for this operon also inhibits transcription initiation at $\mathrm{P}_{\text {epeAl }}$ (Fig. 3c). This regulation was confirmed in the spo0A mutant, in which $\mathrm{P}_{\text {epeA1 }}$ activity remained constantly repressed throughout the experiment due to the lack of Spo0A-dependent AbrB repression (Fig. 3c). Remarkably, we could also demonstrate a strong constitutive promoter activity for $\mathrm{P}_{\text {epeA2}}$, a fragment lacking the $\mathrm{P}_{\text {epeA1 }}$ sequence. Here, we observed mutual regulation of $\mathrm{AbrB}$ and Spo0A only in late stationary phase (Fig. 3d).

In contrast, the internal control fragment, a random sequence from within $e p e B$, showed no relevant luminescence signal above background (Fig. 3a), demonstrating that all our cloned promoter fragments indeed represent true promoters. Overall, the promoter activities for both $\mathrm{P}_{\text {epeX }}$ and $\mathrm{P}_{\text {epeA }}$ show comparable strength at peak levels as the constitutive and well-characterized Bacillus promoter $\mathrm{P}_{\text {lep } A}$. Note that the slight and transient "peak" of luminescence signal that is observed for all three $\mathrm{P}_{\text {epeA }}$ fragments and the negative control fragment at the transition from exponential growth to stationary phase $(\mathrm{t}=\mathrm{ap}$ prox. $3 \mathrm{~h}$ ) is a known artifact from using the luxABCDEreporter, which can be observed for any promoter fragment [Radeck et al., 2013; Popp et al., 2017].

Taken together, the epe locus in B. subtilis is transcribed from three independent promoters, $\mathrm{P}_{\text {epeX }}, \mathrm{P}_{\text {epeAl }}$, and $\mathrm{P}_{\text {epeA2 }}$. Expression from $\mathrm{P}_{\text {epeX }}$ is strongly induced at the onset of stationary phase, since increasingly active Spo0A relieves the epeX promoter region from AbrB repression, based on strongly and abundantly binding to it during exponential phase. This de-repression results in a full-length transcript covering epeXEPAB. $\mathrm{P}_{\text {epeA1 }}$ and $\mathrm{P}_{\text {epeA2}}$, on the other hand, lack this tight growth phase dependence and presumably ensure steady levels of the immunity transporter EpeAB prior to EpeX* production. A mutational study of strains lacking stationary phase master regulators demonstrated that $\mathrm{AbrB}$ is directly involved in maintaining low transcription of the epe locus, presumably by tightly binding across the complete epe locus [Chumsakul et al., 2013].

\section{EpeAB and LiaIH Provide Autoimmunity against Intrinsically Produced Active EpeX*}

After demonstrating the phylogenetic distribution of the epe locus and studying its expression and regulation, we next investigated the production of EpeX* and its intrinsic antimicrobial efficiency against $B$. subtilis itself. Earlier studies, based on a saturating transposon mutagenesis, indicated that the gene products of epe $A B$ provide intrinsic immunity against EpeX: disruptions of either gene resulted 
Fig. 4. Intrinsic EpeX production and resistance. a Xylose-dependent (over)production of EpeX* from an inducible copy of epeXEP integrated into the lacA locus. Growth curves of the wild type and isogenic epe $A B$, liaIH mutants are depicted. In the absence of xylose, no growth effects were observed between the strains (left panel). Upon induction of the extra copy of epeXEP by addition of $1 \%$ xylose after $1 \mathrm{~h}$ (black line), the combined deletion of epe $A B$ and liaIH caused a severe growth defect (right panel). $\mathbf{b}$ Mass spectrometry analysis of $B$. subtilis supernatants derived from the wild type and epe locus deletion strains carrying xylose-inducible copies of either epeXEP or epeXE. The EpeX concentrations in $\mathrm{nM}$, normalized to the optical densities of respective strains, are shown after xylose addition (suppl. Fig. 3). All data shown in a derive from biological duplicates and technical triplicates. Measurements in b were performed in biological and technical duplicates.
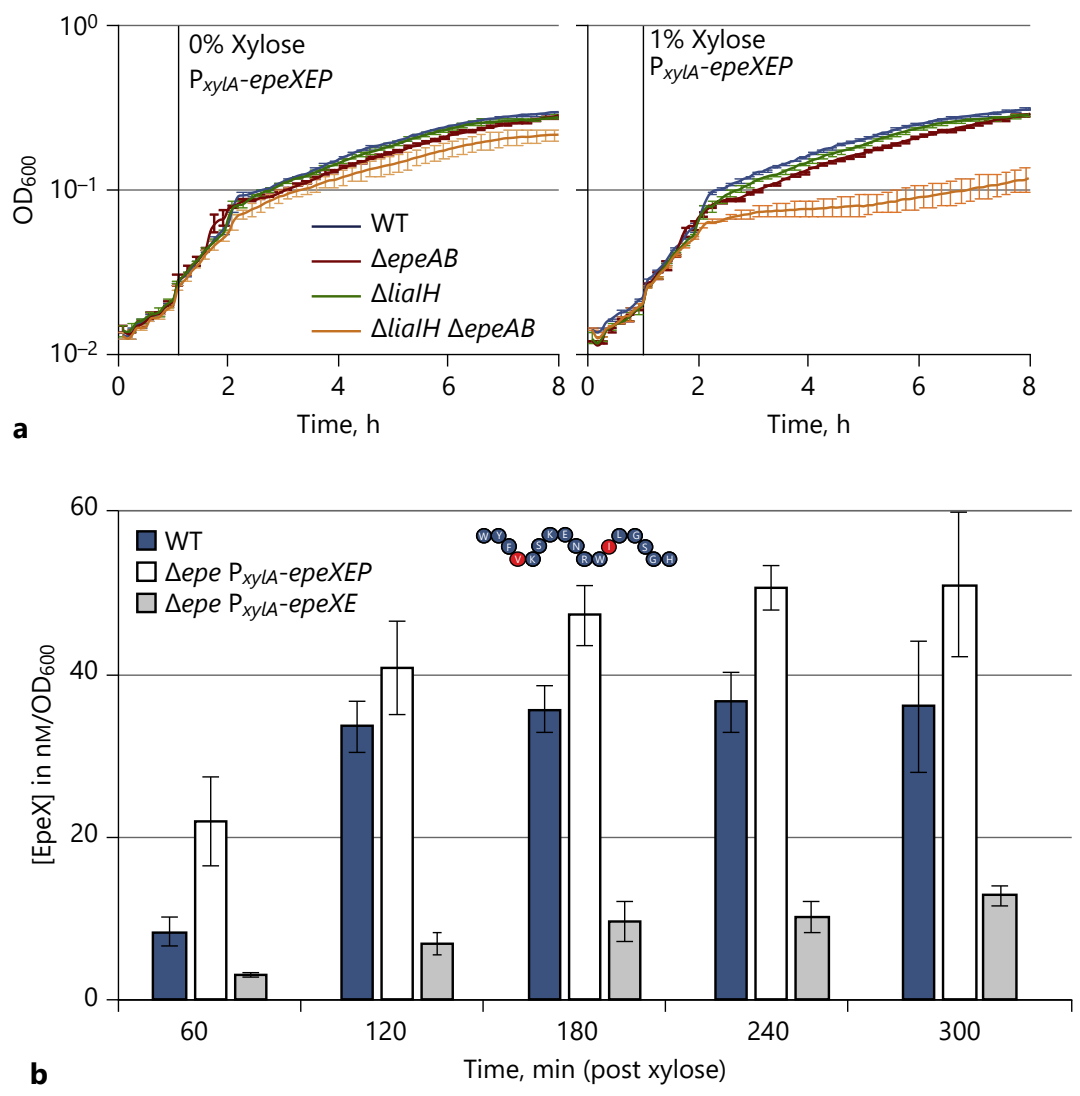

in the induction of the Lia-dependent envelope stress response, indicating that in the absence of this $\mathrm{ABC}$ transporter, the membrane of $B$. subtilis is somehow perturbed [Butcher and Helmann, 2006]. More recently, we verified and expanded on these initial observations by demonstrating that deletions of either epeA or epeB intrinsically triggered the Lia response without any impairment of growth [Popp et al., 2020]. We could also demonstrate that the target operon of the Lia response, liaIH, provides resistance against extrinsically applied EpeX* [Popp et al., 2020].

Here, we further elucidated the role of the Lia system in providing resistance against EpeX* by investigating the effects of overproducing the core biosynthetic genes, epeXEP (Fig. 1) from an additional xylose-inducible copy in the wild type and isogenic mutant strains lacking epe$A B$, liaIH, or both. We monitored growth over time and induced production of the extra epeXEP copy after $1 \mathrm{~h}$ with $1 \%$ xylose (Fig. 4a, right panel). Under non-inducing conditions, no growth differences were observed (Fig. 4a, left panel). In contrast, approximately $1 \mathrm{~h}$ after inducing epeXEP expression from the extra xylose-inducible copy ( $\sim 2 \mathrm{~h}$ into the experiment), we observed a severe growth defect of the epeAB/liaIH double mutant, while both single mutants were indistinguishable from the wild type. It is important to note that all strains still contained the native epe locus. We conclude from these data that increasing the amount of epeXEP alone can cause physiological consequences that are then counteracted by either EpeAB or LiaIH. Each resistance determinant can compensate for the other under these experimental conditions and ensure normal growth even in the presence of increased EpeX levels. In contrast, the lack of both resistance determinants at the same time cannot be tolerated and cells become damaged by EpeX* (Fig. 4a, right panel).

\section{Export of Active EpeX* Requires the Membrane- Anchored Peptidase EpeP}

The results described above not only illuminated the role the Lia system has in contributing to the resistance against intrinsically produced EpeX*, but they also demonstrated that expression of epeXEP from a xylose-inducible promoter leads to an increase in the production of the active EpeX* peptide. This allowed uncoupling the production of this bacteriocin from the complex growth phase- 
dependent regulation described further above, thereby enabling to further elucidate the contribution of all genes encoded in the epeXEPAB locus to the biosynthesis of active EpeX*. The essential role of EpeE in modifying EpeX has already been studied in mechanistic detail [Benjdia et al., 2017a; Benjdia et al., 2017b]. The results described in the previous section not only supported the role of EpeAB in autoimmunity, they also further argue against a role of this $\mathrm{ABC}$ transporter in EpeX export. In contrast, the role of the proposed membrane-anchored peptidase EpeP has so far not been addressed experimentally. Based on its domain architecture, it is attractive to postulate that it performs both export and proteolytic processing simultaneously, thereby releasing the active, post-translationally modified 17-mer EpeX*. This allowed drawing a testable hypothesis: If Epe $A B$ is indeed not involved in EpeX export, and if this role is exclusively provided by EpeP, then the functions encoded by epeXEP are necessary and sufficient for producing the active extracellular AMP EpeX*, while epeXE alone would not suffice. As demonstrated both above (Fig. 4a) and previously [Butcher, and Helmann, 2006; Popp et al., 2020], loss of the autoimmunity function of EpeAB can be tolerated and compensated for by LiaIH.

We therefore determined the total amount of exported EpeX* in the supernatants of B. subtilis wild type and engineered strains in which the native epe locus has been completely removed and complemented with an ectopic copy of either epeXEP or epeXE under a xylose-inducible promoter. This setup enabled us to (i) determine the amounts of EpeX*, (ii) compare EpeX* concentrations between the wild type and the xylose-regulated copies and thereby (iii) investigate the role of the membranebound EpeP peptidase with respect to its proposed function processing and in exporting active EpeX*.

Respective strains were cultivated in chemical defined medium, and xylose was added in early exponential growth phase followed by subsequent hourly harvesting of supernatants for $5 \mathrm{~h}$ (suppl. Fig. 3 and Material and Methods). EpeX* concentrations were determined by mass spectrometry and amounts were normalized to the optical density of each time point (shown in Fig. $4 \mathrm{~b}$ and Fig. S3). We were able to identify the 17-mer epimerized form of EpeX* and determined at the first time point about $10 \mathrm{nM}$ for the wild type and $20 \mathrm{nM}$ for the strain harboring $\mathrm{P}_{x y l A^{-}}$epeXEP. At all subsequent time points, both of these strains yielded comparable EpeX* amounts between 35 and $50 \mathrm{nM}$, with the xylose-inducible copy of epeXEP always producing slightly higher amounts of EpeX*, probably due to the differences in regulation or promoter dynamics (Fig. 4b). For the wild type, we could

Epipeptide Biosynthesis Locus of $B$. subtilis observe a growth phase-dependent increase in exported EpeX*, reaching its peak at the onset of stationary phase (Fig. 4b, 240 min, and Fig. S3). This dynamic is in agreement with the AbrB-Spo0A-dependent regulation of the native epeXEPAB locus. While the $\mathrm{P}_{x y l A}$-epeXEP construct could therefore complement the epeXEPAB deletion, introduction of epeXE alone was not sufficient, as hypothesized. Nevertheless, and surprisingly, we were still able to detect low amounts (between three and $13 \mathrm{nM}$ ) of EpeX* even in the absence of EpeP (Fig. 4b). This significant (over 5-fold) decrease in AMP concentrations demonstrates a crucial role of the EpeP peptidase in the biosynthesis and export of active EpeX* for the first time. The low but detectable AMP amounts in the absence of EpeP indicate that $B$. subtilis has additional mechanisms to process and export the modified pre-peptide. These could be based on a so far unknown alternative export mechanism for EpeX or, more likely, lysed cells. Future studies need to clarify if secreted peptidases of $B$. subtilis are potentially also capable of cleaving pre-EpeX [McAuliffe et al., 2001].

\section{Discussion}

Many bacteria, including B. subtilis, produce secondary metabolites to survive in complex and competitive habitats such as the soil [Kaspar et al., 2019]. Here, we addressed a number of open questions regarding the distribution, expression, and regulation of the minimalistic epeXEPAB locus of $B$. subtilis, as well as the biosynthesis and biological efficiency of the produced epipeptide EpeX*, a ribosomally synthesized and post-translationally modified AMP. We demonstrated that the epe locus is restricted to and widely distributed in individual genomes of the phylum Firmicutes, without showing any preference to certain genera. In the genomes harboring the epe locus, the sequence of the EpeX pre-pro-peptide is particularly well conserved in the C-terminal 17 -mer region of the mature EpeX* and still well-conserved for the central third, which is postulated to contain the recognition sequence for the membrane-anchored EpeP peptidase, which mediates the processing and export of EpeX* (Fig. 2). The gene products of epeXEP are necessary and sufficient for producing wild-type amounts of mature EpeX* (Fig. 4). The epeXEPAB locus is expressed from three promoters, an AbrB-dependent and thereby Spo0Acontrolled promoter upstream the epe locus $\left(\mathrm{P}_{\text {epeX }}\right)$ and two promoters inside the coding sequence of epeP, which ensures constitutive expression of epe $A B$ and hence pre- 
sumably autoimmunity prior to EpeX* production $\left(\mathrm{P}_{\text {epeA1 }}\right.$ and $\left.\mathrm{P}_{\text {epeA2 }}\right)$ (Fig. 3).

Previously, we have shown that EpeX*, when applied externally in $\mu \mathrm{M}$ concentrations, inhibits growth of $B$. subtilis by severely perturbing the cytoplasmic membrane. Under these experimental conditions, the general envelope stress proteins $\mathrm{LiaIH}$, but not the autoimmunity transporter EpeAB, provided some degree of protection against EpeX* [Popp et al., 2020]. In this report, we could demonstrate that LiaIH and EpeAB act together and can compensate for each other - to protect cells from intrinsically produced EpeX* (Fig. 4a). Most remarkably, the concentration of free EpeX* in the supernatant of the wild type was in the nM range (Fig. $4 \mathrm{~b}$ ), that is, three orders of magnitude lower than what has been externally applied previously. How can this apparent contradiction be resolved? The most obvious explanation is to propose that neither the externally applied $\mu \mathrm{M}$ EpeX* concentration nor the nM EpeX* concentrations measured in the supernatant of producing cultures (Fig. $4 \mathrm{~b}$ ) provide a reliable, that is, physiologically meaningful estimate of the EpeX* fraction in the culture that is biologically active the EpeX* molecules perturbing the integrity from the outside face of the cytoplasmic membrane. In the case of externally applied EpeX*, it is possible that the vast majority of the AMP is retained by e.g., the cell wall and hence never reaches its target site. In case of exported EpeX, the local concentration of biologically active EpeX* may be much higher directly at the outside face of the cytoplasmic membrane of producing cells than what is ultimately released to (and hence can be detected in) the culture supernatant. Accordingly, the vast concentration differences between externally applied $(\mu \mathrm{M})$ and released (nM) EpeX* in B. subtilis cultures might be dramatically smaller at the site of action. Experimentally addressing this difference will be challenging and require approaches that are clearly beyond the scope of this report.

Irrespective of the local and biologically relevant EpeX* concentration, a second striking difference has been observed between the previous report [Popp et al., 2020] and the data reported herein: While LiaIH was the only resistance determinant against externally applied EpeX*, LiaIH and EpeAB together provided protection against self-produced EpeX* and can compensate for each other (Fig. 4a). Why does the $\mathrm{ABC}$ transporter EpeAB provide resistance against self-produced but not externally applied EpeX*? One possibility is that the autoimmunity function of Epe$\mathrm{AB}$ is tightly linked to the exporting/processing action of EpeP. Based on the results presented in this study, EpeAB is presumably produced prior to EpeX* release, based on the constitutive epeAB transcription from $\mathrm{P}_{e p e A 1}$ and/or $\mathrm{P}_{\text {epeA2 }}$ already during exponential growth phase (Fig. 3b). Accordingly, EpeAB is already present in the membrane to directly take care of EpeX* upon release by EpeP, thereby ensuring that autoimmunity is sufficient to protect the cells from their self-produced toxin. Such a tight association of EpeX* sensing by EpeAB to EpeP-dependent release would explain, why EpeAB cannot provide resistance to externally applied EpeX*, which reaches the outside face of the cytoplasmic membrane independent of EpeP.

Induction of LiaIH production is transcriptionally coordinated by the damage-sensing three-component system LiaFSR [Jordan et al., 2006; Wolf et al., 2012], which responds to envelope stress caused by the action of antimicrobial compounds that either interfere with membrane-anchored steps of cell wall biosynthesis, such as bacitracin or vancomycin [Mascher et al., 2004], or membrane integrity, such as daptomycin or EpeX* [Wecke et al., 2009; Popp et al., 2020]. This sensing is based on the envelope damage caused by these compounds, but independent of their chemical nature, mode of action or mechanism of biosynthesis. Accordingly, the Lia system can respond to and mediate resistance against both externally applied and self-produced EpeX*. In this scenario, the Lia response is therefore a back-up system that provides a secondary layer of protection if the production (or at least the external concentration) of EpeX* surpasses the capacity of the autoimmunity system. This function would be comparable to what has been shown for its role in bacitracin resistance [Radeck et al., 2016a; Radeck et al., 2016b].

So far, the link between Lia-dependent envelope stress response and production of the AMP EpeX* has been primarily based on the latter causing membrane perturbations that are sensed by the former [Popp et al., 2020]. The data presented in this report also provide an additional regulatory link between the expression of the epeXEPAB and the liaIH operons. Both are under direct control of the transition state repressor $\mathrm{AbrB}$ and thereby indirectly controlled by the activity of the sporulation master regulator Spo0A (Fig. 3a and [Jordan et al., 2007]). When the population enters stationary phase, expression of $\mathrm{AbrB}$ - which globally suppresses stationary phase function during exponential growth - is repressed by SpoOA, the master regulator governing sporulation in $B$. subtilis [Popp and Mascher, 2019; Lopez et al., 2009; Fujita and Losick, 2005]. The epe locus is a primary AbrB-target with eleven high-affinity $\mathrm{AbrB}$ binding sites mapped across

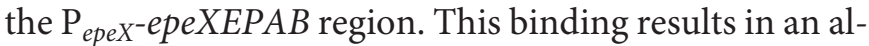
most complete coverage of this genomic region by this transition state repressor (Fig. S2, extracted from [Chum- 
Table 1. Strains used in this study

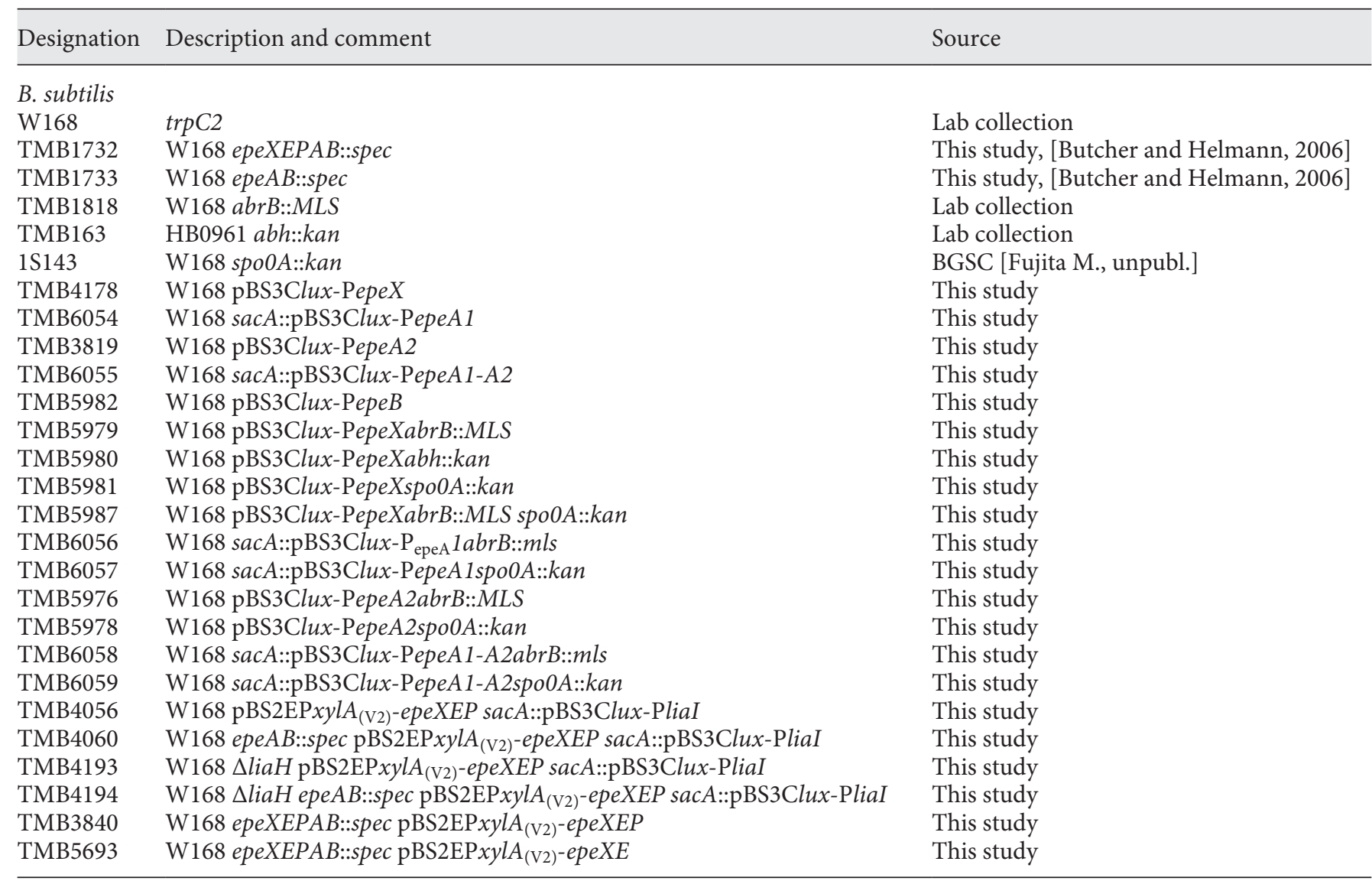

sakul et al., 2013]), thereby ensuring that epe expression (and hence EpeX* production) is shut off during exponential growth. In contrast, a single low-affinity AbrB binding site was mapped by the same study in the intergenic region upstream the liaIH operon and verified independently (Fig. S2, extracted from [Chumsakul et al., 2013]; [Jordan et al., 2007]). Induction of the liaIH operon is therefore dually affected by AbrB: indirectly through a massive repression of the epe operon during exponential phase and directly through interference of AbrB with LiaR-binding to its target promoter, $\mathrm{P}_{\text {lial }}$ ([Jordan et al., 2007] and this study).

In summary, this study answered a number of important questions regarding epeXEPAB expression and regulation, as well as production of and resistance against intrinsically produced EpeX*. The most important open question remaining concerns the biological function of this epipeptide. Its narrow host range and high efficiency against B. subtilis, as well as its Spo0A-dependent expression point towards a potential function of EpeX* as a third cannibalism-like toxin in the differentiation of $B$. subtilis rather than a role as a defense mechanism against microbial competitors [Popp and Mascher, 2019]. So far, this is only a hypothesis that needs to be addressed by future studies in order to reveal the true physiological role of EpeX*.

\section{Materials and Methods}

Bacterial Strains and Growth Conditions

B. subtilis and Escherichia coli were grown at $37^{\circ} \mathrm{C}$ with aeration (at $200 \mathrm{rpm}$ agitation) in one of the following media: (i) Lysogeny broth (LB medium) or chemical defined medium (ii) MNGE [88.2\% $1 \times \mathrm{MN}$ medium $\left(1.36 \%(\mathrm{w} / \mathrm{v})\right.$ dipotassium phosphate $\times 3 \mathrm{H}_{2} \mathrm{O}$, $0.6 \%(\mathrm{w} / \mathrm{v})$ monopotassium phosphate, $0.1 \%(\mathrm{w} / \mathrm{v})$ sodium citrate $\times$ $\left.\mathrm{H}_{2} \mathrm{O}\right), 1.9 \%$ glucose, $0.19 \%$ potassium glutamate, $0.001 \%(\mathrm{w} / \mathrm{v}) \mathrm{am}-$ monium ferric citrate, $0.005 \%(\mathrm{w} / \mathrm{v})$ tryptophan, and $0.035 \%(\mathrm{w} / \mathrm{v})$ magnesium sulfate]. For solid agar plates, $1.5 \%(\mathrm{w} / \mathrm{v})$ agar-agar was added. All strains used in this study are listed in Table 1. B. subtilis cells carrying a resistance marker were selected using chloramphenicol $\left(5 \mu \mathrm{g} \mathrm{mL}{ }^{-1}\right)$, kanamycin $\left(10 \mu \mathrm{gL}^{-1}\right)$, or erythromycin com- 
bined with lincomycin $\left(1 \mu \mathrm{g} \mathrm{mL} L^{-1}, 25 \mu \mathrm{g} \mathrm{mL} L^{-1}\right)$ for MLS. Transformation of E. coli and B. subtilis was performed as described previously [Harwood and Cutting, 1991; Sambrook and Russel, 2001].

\section{DNA Manipulation}

Plasmids were constructed using standard cloning techniques as described elsewhere [Sambrook, and Russell, 2001]. For DNA amplification via PCR, Q $5{ }^{\circledR}$ polymerase was used. Enzymes were purchased from New England Biolabs (NEB, Ipswich, MA, USA) and applied following their respective protocols. Positive E. coli clones were checked by colony PCR, using OneTaq ${ }^{\circledR}$ polymerase. All constructs were verified by sequencing. Successful integration of the plasmids into the B. subtilis genome were confirmed via colony PCR of respective up and down fragments. All primers and plasmids used in this study are listed in suppl. Tables S2 and S3.

\section{Bioinformatics Analysis}

To investigate the phylogenetic distribution of the epipetide EpeX, a PSI-blast [Altschul et al., 1997; Schäffer et al., 2001] search with four iterations based on the B. subtilis EpeX protein sequence (NP_391898.1) was performed. In total, 49 sequences were obtained applying the default parameters of the PSI-blast algorithm (suppl. Table 1). From these sequences, 33 next to the query sequence were found in the MiST3 database [Gumerov et al., 2020]. From these 34 proteins, a phylogenetic tree was computed using TREND [Gumerov and Zhulin, 2020], applying the maximum likelihood and bootstrap (100) method. Sequence redundancy was minimized using the integrated CD-hit method (cut-off value 0.9) resulting in a final dataset of 19 sequences (suppl. Table 1 and shown in Fig. 2) [Huang et al., 2010]. The multiple sequence alignment of these proteins was performed applying MAFFT [Katoh et al., 2017] and regions of low conservations if any were deleted in Jalview [Waterhouse et al., 2009]. Finally, a weblogo was generated using the browser-based version of WebLogo [Crooks et al., 2004].

\section{Luciferase Assay}

A detailed description of the procedure was reported previously [Popp et al., 2017; Radeck et al., 2013]. In brief, overnight cultures were grown in LB with respective antibiotics. Day cultures, without antibiotics, were inoculated 1:200 in freshly made prewarmed medium as indicated and grown until an $\mathrm{OD}_{600}$ of $0.1-0.4$. Subsequently, the cells were diluted to an $\mathrm{OD}_{600}$ of 0.01 . Then, 100 $\mu \mathrm{L}$ of cells per sample were transferred into a 96-well plate (black walls, clear bottom, Greiner Bio-One, Frickenhausen, Germany). If appropriate, after $1 \mathrm{~h}$, the cells were induced with xylose to the indicated final concentrations. The assay was performed using a SynergyTM NeoalphaB microtiter platereader (BioTek, Winooski, VT, USA). The optical density as well as luminescence was measured every $5 \mathrm{~min}$ for at least $15 \mathrm{~h}$.

\section{Determination of Exported EpeX Peptide}

All strains were grown in MNGE medium, supplemented with respective antibiotics. A freshly grown culture was used to inoculate $100 \mathrm{~mL}$ of MNGE medium. Growth proceeded at $37^{\circ} \mathrm{C}$ and 200 rpm. Xylose ( $1 \%$ final) was added when $\mathrm{OD}_{600} \mathrm{~nm}$ reached 0.2 . Samples $(250 \mu \mathrm{L})$ were centrifugated at room temperature for $5 \mathrm{~min}$ at $14,000 \mathrm{rpm} \mathrm{min}{ }^{-1}$, and the supernatant was frozen with liquid nitrogen. Each experiment was repeated twice independently. For detection of the EpeX peptide (17mer), $2 \mu \mathrm{L}$ of supernatant was injected on a Gold C18 AQ column $(2 \times 200 \mathrm{~mm}, 1.9 \mu \mathrm{m})$, at a flow rate of $0.45 \mathrm{~mL} \mathrm{~min}^{-1}$ at $55^{\circ} \mathrm{C}$. Elution was performed with an acetonitrile gradient containing $0.1 \%$ formic acid during 8.5 min. Detection was performed with an HESI2 probe connected to a Q-Exactive Focus mass spectrometer. Single ion monitoring mode (m/z range: 525.62-528.62) was used to detect the EpeX (WYFVKSKENRWILGSGH) peptide. Peptide mass was determined with 6 ppm accuracy. Peptide sequence was identified with $\mathrm{X}$ !tandemPipeline (version XTPcpp 0.2.16) ran against the Uniprot $B$. subtilis 168 database and manually validated.

\section{Data Analysis and Statistical Procedures}

Growth and luminescence measurements were performed in biological and technical duplicates or triplicates. From the values obtained for each time point, mean values and standard deviation $(+/-)$ were calculated and plotted (shown in Fig. 3, 4a and suppl. Fig. 3). Analysis of mass spectrometry to determine the concentration of exported EpeX peptide in B. subtilis supernatants, provided by Benjdia. A. and Berteau, O. from the Université Paris-Saclay, INRAE, AgroParisTech, Micalis Institute, was performed in biological duplicates (shown in Fig. 4b and suppl. Fig. 3).

\section{Acknowledgement}

The authors want to thank all current and past members of the Mascher lab for helpful discussions and feedback. Moreover, we would like to acknowledge Ulrike Mäder for providing the promoter sequence for $\mathrm{P}_{\text {epeAl }}$ shown in Figure 1c.

\section{Statement of Ethics}

No ethical approval is required since no human studies or studies involving animals were conducted.

\section{Conflict of Interest Statement}

The authors have no conflicts of interest to declare.

\section{Funding Sources}

This work was supported by a grant from the Deutsche Forschungsgemeinschaft (MA2837/3 to T.M.) in the framework of the priority program SPP1617 "Phenotypic heterogeneity and sociobiology of bacterial populations" and by the European Research Council ERC consolidator Grant 617053 (to O. B.). Open Access Funding was provided by the research training group GRK1708 "Molecular Principles of Bacterial Survival Strategies."

\section{Author Contributions}

P.F.P and T.M. planed the project. P.F.P., L.F., A.B., A.G., O.B., conducted the experiments. P.F.P., T.M., L.F., A.B., A.G., O.B., analyzed and interpreted the data. P.F.P. and T.M. wrote the manuscript. 


\section{References}

Altschul SF, Madden TL, Schäffer AA, Zhang J, Zhang Z, Miller W, et al. Gapped BLAST and PSI-BLAST: a new generation of protein database search programs. Nucleic Acids Res. 1997 Sep 1;25:3389-402.

Benjdia A, Guillot A, Ruffié P, Leprince J, Berteau O. Post-translational modification of ribosomally synthesized peptides by a radical SAM epimerase in Bacillus subtilis. Nat Chem. 2017a Jul;9:698-707.

Benjdia A, Balty C, Berteau O. Radical SAM Enzymes in the Biosynthesis of Ribosomally Synthesized and Post-translationally Modified Peptides (RiPPs). Front Chem. 2017b;5: 87.

Butcher BG, Helmann JD. Identification of Bacillus subtilis sigma-dependent genes that provide intrinsic resistance to antimicrobial compounds produced by Bacilli. Mol Microbiol. 2006 May;60:765-82.

Chumsakul O, Nakamura K, Kurata T, Sakamoto T, Hobman JL, Ogasawara N, et al. High-resolution mapping of in vivo genomic transcription factor binding sites using in situ DNase I footprinting and ChIP-seq. DNA Res. 2013 Aug;20:325-38.

Cotter PD, Hill C, Ross RP. Bacteriocins: developing innate immunity for food. Nat Rev Microbiol. 2005 Oct;3:777-88.

Crooks GE, Hon G, Chandonia J-M, Brenner SE. WebLogo: a sequence logo generator. Genome Res. 2004 Jun;14:1188-90.

Czárán TL, Hoekstra RF, Pagie L. Chemical warfare between microbes promotes biodiversity. Proc Natl Acad Sci U S A. 2002 Jan 22;99:78690.

Finking R, Marahiel MA. Biosynthesis of nonribosomal peptides1. Annu Rev Microbiol. 2004;58:453-88.

Foster KR, Bell T. Competition, not cooperation, dominates interactions among culturable microbial species. Curr Biol. 2012 Oct 9;22: 1845-50.

Fujita M, Losick R. Evidence that entry into sporulation in Bacillus subtilis is governed by a gradual increase in the level and activity of the master regulator Spo0A. Genes Dev. 2005 Sep 15;19:2236-44

Gumerov VM, Ortega DR, Adebali O, Ulrich LE, Zhulin IB. MiST 3.0: an updated microbial signal transduction database with an emphasis on chemosensory systems. Nucleic Acids Res. 2020 Jan 8;48:D459-64.

Gumerov VM, Zhulin IB. TREND: a platform for exploring protein function in prokaryotes based on phylogenetic, domain architecture and gene neighborhood analyses. Nucleic Acids Res. 2020 Jul 2;48:W72-6.
Harwood CR, Cutting SM. Molecular Biological Methods for Bacillus. London: Wiley; 1991.

Huang Y, Niu B, Gao Y, Fu L, Li W. CD-HIT Suite: a web server for clustering and comparing biological sequences. Bioinformatics. 2010 Mar 1;26:680-2.

Jordan S, Junker A, Helmann JD, Mascher T. Regulation of LiaRS-dependent gene expression in Bacillus subtilis: identification of inhibitor proteins, regulator binding sites, and target genes of a conserved cell envelope stress-sensing two-component system. J Bacteriol. 2006 Jul; 188:5153-66

Jordan S, Rietkötter E, Strauch MA, Kalamorz F, Butcher BG, Helmann JD, et al. LiaRS-dependent gene expression is embedded in transition state regulation in Bacillus subtilis. Microbiology (Reading, Engl). 2007 Aug; 153: 2530-40.

Kaspar F, Neubauer P, Gimpel M. Bioactive Secondary Metabolites from Bacillus subtilis: A Comprehensive Review. J Nat Prod. 2019;82: 2038-53.

Katoh K, Rozewicki J, Yamada KD. MAFFT online service: multiple sequence alignment, interactive sequence choice and visualization. Brief Bioinformatics. 2017 Sep 6;30:30591166.

Kerr B, Riley MA, Feldman MW, Bohannan BJM. Local dispersal promotes biodiversity in a real-life game of rock-paper-scissors. Nature. 2002 Jul 11;418:171-4.

Kumariya R, Garsa AK, Rajput YS, Sood SK, Akhtar N, Patel S. Bacteriocins: Classification, synthesis, mechanism of action and resistance development in food spoilage causing bacteria. Microb Pathog. 2019 Mar;128: $171-7$.

Lopez D, Vlamakis H, Kolter R. Generation of multiple cell types in Bacillus subtilis. FEMS Microbiol Rev. 2009 Jan;33:152-63.

Lopez D, Kolter R. Extracellular signals that define distinct and coexisting cell fates in Bacillus subtilis. FEMS Microbiol Rev. 2010 Mar 1; 34:134-49.

Mascher T, Zimmer SL, Smith T-A, Helmann JD. Antibiotic-inducible promoter regulated by the cell envelope stress-sensing two-component system LiaRS of Bacillus subtilis. Antimicrob Agents Chemother. 2004 Aug;48: 2888-96.

McAuliffe O, Ross RP, Hill C. Lantibiotics: structure, biosynthesis and mode of action. FEMS Microbiol Rev. 2001 May;25:285-308.

Nicolas P, Mäder U, Dervyn E, Rochat T, Leduc A, Pigeonneau N, et al. Condition-dependent transcriptome reveals high-level regulatory architecture in Bacillus subtilis. Science. 2012 Mar 2;335:1103-6.
Popp PF, Dotzler M, Radeck J, Bartels J, Mascher T. The Bacillus BioBrick Box 2.0: expanding the genetic toolbox for the standardized work with Bacillus subtilis. Sci Rep. 2017 Nov 8;7: 15058.

Popp PF, Mascher T. Coordinated Cell Death in Isogenic Bacterial Populations: Sacrificing Some for the Benefit of Many? J Mol Biol. 2019 Nov;431(23):4656-4669.

Popp PF, Benjdia A, Strahl H, Berteau O, Mascher T. The Epipeptide YydF Intrinsically Triggers the Cell Envelope Stress Response of Bacillus subtilis and Causes Severe Membrane Perturbations. Front Microbiol. 2020;11:151.

Radeck J, Kraft K, Bartels J, Cikovic T, Dürr F, Emenegger J, et al. The Bacillus BioBrick Box: generation and evaluation of essential genetic building blocks for standardized work with Bacillus subtilis. J Biol Eng. 2013;7:29.

Radeck J, Gebhard S, Orchard PS, Kirchner M, Bauer S, Mascher T, et al. Anatomy of the bacitracin resistance network in Bacillus subtilis. Mol Microbiol. 2016a Jan 27;100:607.

Radeck J, Fritz G, Mascher T. The cell envelope stress response of Bacillus subtilis: from static signaling devices to dynamic regulatory network. Curr Genet. 2016b Jun 25;63:79-90.

Sambrook J, Russell DW. Molecular Cloning. Cold Spring Harbor: CSHL Press; 2001.

Schäffer AA, Aravind L, Madden TL, Shavirin S, Spouge JL, Wolf YI, et al. Improving the accuracy of PSI-BLAST protein database searches with composition-based statistics and other refinements. Nucleic Acids Res. 2001 Jul 15;29:2994-3005.

Strauch M, Webb V, Spiegelman G, Hoch JA. The SpoOA protein of Bacillus subtilis is a repressor of the abrB gene. Proc Natl Acad Sci USA. 1990 Mar;87:1801-5.

Strieker M, Tanović A, Marahiel MA. Nonribosomal peptide synthetases: structures and dynamics. Curr Opin Struct Biol. 2010 Apr;20 $234-40$.

Waterhouse AM, Procter JB, Martin DMA, Clamp M, Barton GJ. Jalview Version 2 - a multiple sequence alignment editor and analysis workbench. Bioinformatics. 2009 May 1; 25:1189-91.

Wecke T, Zühlke D, Mäder U, Jordan S, Voigt B, Pelzer S, et al. Daptomycin versus Friulimicin B: in-depth profiling of Bacillus subtilis cell envelope stress responses. Antimicrob Agents Chemother. 2009 Apr;53:1619-23.

Wolf D, Domínguez-Cuevas P, Daniel RA, Mascher T. Cell envelope stress response in cell wall-deficient L-forms of Bacillus subtilis. Antimicrob Agents Chemother. 2012 Nov;56: 5907-15. 